\title{
Dynamic phase reversal of photo-induced precession of magnetization in ferromagnetic
}

\section{(Ga,Mn)As thin film}

\author{
Hang Li and Xinhui Zhang ${ }^{\text {a) }}$
}

State Key Laboratory of Superlattices and Microstructures, Institute of Semiconductors, Chinese Academy of Sciences, P.O. Box 912, Beijing 100083, P. R. China

Xinyu Liu and Jacek K. Furdyna

Department of Physics, University of Notre Dame, Notre Dame, Indiana 46556, USA

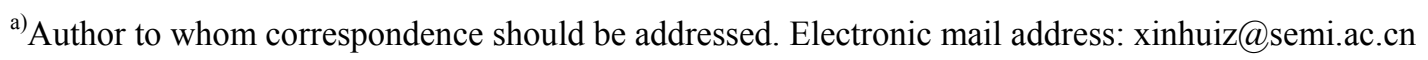




\begin{abstract}
Ultrafast laser-triggered coherent magnetization dynamics in ferromagnetic $(\mathrm{Ga}, \mathrm{Mn}) \mathrm{As}$ films has been investigated by time-resolved magneto-optical spectroscopy. Dynamic phase reversal in the magnetic precession process is observed when the ambient temperature or the external magnetic field is varied. This phenomenon is found to be sensitive to the spontaneous magnetization orientation, and is attributed to the giant magnetic linear dichroism (MLD) effect in $(\mathrm{Ga}, \mathrm{Mn})$ As. Our findings suggest that this effect will enable the sensitive measurement of the dynamic phase of in-plane magnetization precession on picosecond time scale in the collective spin excitation in $(\mathrm{Ga}, \mathrm{Mn}) \mathrm{As}$, thus enabling efficient and ultrafast magneto-optical detection for magnetization dynamics in ferromagnetic semiconductor-based spintronic devices.
\end{abstract}




\section{Introduction}

Ultrafast magneto-optical investigation of III-V Mn-based ferromagnetic semiconductors has drawn much interest in simultaneous control of both magnetic and semiconductor properties of these materials from the point of view of the fundamental physics and of spintronic applications [1-4]. Even though mechanism for hole-mediated magnetization dynamics in the ferromagnetic semiconductor $(\mathrm{Ga}, \mathrm{Mn})$ As involving the $s p-d$ exchange coupling between the itinerant carriers and localized Mn spins is not yet fully understood [5-11], a variety of ultrafast pump-probe magneto-optical phenomena already observed in this material demonstrate the possible multi-functionality of ferromagnetic semiconductors when incorporated in spintronic devices[10, 12-15]. Recent studies of magnetization dynamics in $(\mathrm{Ga}, \mathrm{Mn})$ As were primarily focused on understanding the physical mechanisms of ultrafast excitation and magnetic precession damping in this material $[10,14]$. However, regardless of the kind of excitation mechanisms is applied, the understanding of the specific ultrafast response of magnetization upon external modulation will be of key importance for high-speed magnetic information storage.

Recently, it has been reported that the three-dimensional magnetization precession trajectory excited by a laser pulse can be reconstructed by disentangling the out-of-plane and the in-plane projections of the magnetization based on magnetic linear dichroism (MLD)and on the polar Kerr effect (PKE) in ferromagnetic (Ga,Mn)As [16-18]. The strong in-plane magneto-optical effect in this material makes the giant MLD a powerful technique for studying hole-mediated ferromagnetism via the quantitative measurement of magnetization precession 
[19]. Besides, MLD can also serve as an efficient experimental tool for investigating materials' properties as well as basic physical phenomena [17]. Although the dependence of magnetic hysteresis on the incident light polarization and the spectral dependence of the MLD response in (Ga,Mn)As have already been studied [16,18], the time-dependent dynamics behavior of the magnetization vector as function of temperature and external magnetic field in (Ga,Mn)As requires further investigation. Here the giant MLD effect can provide essential understanding of this behavior that is key importance for its application in the high-speed magnetic information storage.

In this paper we investigate the laser-triggered coherent magnetization dynamics in ferromagnetic (Ga,Mn)As by time-resolved pump-probe magneto-optical measurements. We find that the measured dependence of laser-induced magnetization precession on ambient temperature and on external magnetic field is accompanied by a clear dynamic phase reversal originating from the MLD effect. Here we use linearly-polarized pump light to exclude possible contributions from photon angular momentum, and the systematic phase reversals in magnetization precession are observed by modulating the equilibrium magnetization orientation either by increasing the temperature or by varying the external field. Our results suggest that phase reversals of the dynamic precession observed in $(\mathrm{Ga}, \mathrm{Mn}) \mathrm{As}$ films directly reflect the instantaneous changes of relationship between the equilibrium magnetization orientation and the polarization direction of the probe beam due to the giant MLD effect, thus providing an efficient optical method for visualizing the dynamic magnetization reorientation in ultrafast collective spin behavior for potential (Ga,Mn)As-based spintronic devices. 


\section{Experimental details}

Ferromagnetic $\mathrm{Ga}_{0.964} \mathrm{Mn}_{0.036} \mathrm{As}$ thin films with thickness of $97 \mathrm{~nm}$ were prepared by low-temperature molecular-beam epitaxy (LT-MBE) on GaAs(001) substrates. A 150nm-thick GaAs buffer was first grown at $600^{\circ} \mathrm{C}$ on a GaAs substrate, followed by a $2.2 \mathrm{~nm}$ GaAs layer and $97 \mathrm{~nm}(\mathrm{Ga}, \mathrm{Mn})$ As grown at low temperature $\left(270^{\circ} \mathrm{C}\right)$. The growth was monitored by the reflection high-energy electron diffraction (RHEED) during the entire growth process. The resulting samples were examined by X-ray diffraction (XRD) to confirm crystal quality of the film: no second phases were observed in the (Ga,Mn)As film (i.e., the uniform composition, smooth interfaces, and a high quality single crystalline structure).

The magnetization of the $(\mathrm{Ga}, \mathrm{Mn})$ As film lies in-plane because of the compressive strain. Before optical studies, the sample was annealed at $286^{\circ} \mathrm{C}$ for 1 hour in $\mathrm{N}_{2}$ to enhance the Curie temperature up to $\sim 80 \mathrm{~K}$, as measured by a superconducting quantum interference device (SQUID) magnetometer. In this study, the external magnetic field was applied along the [1 $\overline{1} 0]$ direction, and no external magnetic field was applied when cooling. Photo-induced magnetization dynamics of Mn spins in the ferromagnetic (Ga,Mn)As film was investigated by time-resolved magneto-optical Kerr effect (TR-MOKE), using a Ti:Sapphire laser with a pulse width of $150 \mathrm{fs}$ and a repetition rate of $80 \mathrm{MHz}$. The pumping energy density per pulse was $2.33 \mu \mathrm{Jcm}^{-2}$, with the polarization of the pump beam adjusted to either circular or linear. The probe beam was set to be linearly-polarized. To exclude the possible extrinsic response, the initial reference phase of the lock-in amplifier was fixed during the entire measurement. Since the MLD effect is sensitive to the density of states in the vicinity of the Fermi level of the 
measured magnetic sample, we set the photon energy to $1.58 \mathrm{eV}$ (i.e., near the energy gap of (Ga,Mn)As) so as to maximize the amplitude of the MLD signal $[16,18,19]$. It is now well established that the compressively-strained $(\mathrm{Ga}, \mathrm{Mn}) \mathrm{As}$ layer as used in our experiment is characterized by an in-plane biaxial magnetocrystalline anisotropy with easy axes along [100] and [010] at low temperatures. The MLD response is known to be maximized when the polarization angle $\beta=45^{\circ}[16-18]$, where $\beta$ is defined as the angle between the [100] direction and the polarization orientation of the linearly-polarized probe beam, we thus fix the orientation of the probe polarization close to $45^{\circ}$ in order to have strong enough MLD response. In our experiment, practically the polarization orientation of probe beam is aligned towards $\sim 40^{\circ}$.

\section{Results and discussions}

The time-resolved magneto-optical responses at $10 \mathrm{~K}$ excited by both the linearly- and circularly- polarized pumping pulses without applying external magnetic field are shown in Fig.1. The total magneto-optical signal is superimposed with the exponentially decaying background and oscillatory response. The oscillatory response disappears when the ambient temperature is close to the Curie temperature of $80 \mathrm{~K}$, confirming their ferromagnetic origin related to the magnetization precession [20-24], while the background response which exists above Curie temperature can be attributed to the nonradiative recombination of photo-generated carriers [25]. The intense responses of carriers upon chirality photoexcitation could influence the total magneto-optical signal. The MO responses excited by right- and left- circularly polarized pump pulses show the opposite phases of the pulse-like background signal due to the well known 
optical selection rule [26], which are superposed on the oscillatory response of magnetization precession, as seen in the inset of Fig.1. Previously, the Kerr responses induced by right- and left- circularly polarized pump have shown different phenomenon. Qi and Rozkotová et al. demonstrated the polarization-dependent response upon opposite-helicity excitations in ferromagnetic (Ga,Mn)As [20,22], whereas Y. Hashimoto et al. reported the polarization-independent Kerr response [6], though the similar polarized carrier response upon optical chirality excitation were also observed in different studies by the same group[15]. As is presently understood, this difference may result from different preparation and growth parameters for samples used in different groups and studies. In order to avoid this polarized-carrier induced change of dynamic phase, which is irrelevant to the ferromagnetic order, we therefore apply linearly polarized pump pulse to excite sample to exclude the polarized carrier response and mainly focus on the magnetic precession response.

The strong dependence of the dynamic magneto-optical response on polarization orientation of the probe beam is observed for the $(\mathrm{Ga}, \mathrm{Mn}) \mathrm{As}$ film as previously reported(see Fig.2) when the polarization angle $\beta$ is adjusted from $55^{\circ}$ to $115^{\circ}$. This indicates that the observed magneto-optical response arises mostly from the MLD effect [16-18], which is extremely sensitive to the difference of the optical refractive indices $\Delta n=n_{/ /}-n_{\perp}$, with $n_{\text {/ }}$ and $n_{\perp}$ corresponding to the refractive index of probe beam for polarization perpendicular and parallel to the magnetization vector M, respectively. According to Refs.17 and 18, the MLD response of MO signal can be well described by the sine function of the angle between the probe polarization and the equilibrium direction of the easy axis: $\Delta \beta=\mathrm{P}_{\mathrm{MLD}} \sin \left[2\left(\varphi_{M}-\beta\right)\right]$, where $\beta$ is the angle 
between the incident beam polarization and the [100] crystallographic direction; $\Delta \beta$ is the detected MO response; And $\varphi_{\mathrm{M}}$ is the angle between the magnetization orientation $\mathrm{M}$ and the [100] direction. The equation above is the description for static magneto-optical signal, whereas the dynamical magneto-optical signal can be fitted well by $\operatorname{MO}(\Delta \mathrm{t})=\mathrm{I}_{1} \cos \left[\omega \mathrm{t}+\Phi\left(\varphi_{M}\right.\right.$, $\beta)] \exp \left(-\mathrm{t} / \tau_{1}\right)+\mathrm{I}_{2} \exp \left(-\mathrm{t} / \tau_{2}\right)$, where $\mathrm{I}_{1}, \omega, \Phi, \tau_{1}$ are the precessional amplitude, frequency, phase, relaxation time; $I_{2}$ and $\tau_{2}$ are the intensity and decay time of the background response. When the polarization orientation of probe beam is fixed at $\beta \sim 40^{\circ}$, the equilibrium orientation of magnetization can play an important role on the magnetization precession via MLD effect. Fig.3

(a) shows a systematic dynamical phase shift when the ambient temperature is increased from $10 \mathrm{~K}$ to $60 \mathrm{~K}$. Compared with $\mathrm{MO}$ response measured at $10 \mathrm{~K}$ and $40 \mathrm{~K}$, the precessional phase is reversed, implying the change of the equilibrium orientation of magnetization. With that in mind, we thus measured the temperature dependent remnant magnetization with magnetic field applied along different in-plane crystalline anisotropy axes, as shown in Fig.3(b), in which the temperature-induced reorientation of the easy axis has been confirmed [27,28]. Specifically, it is observed that the easy axis points along the cubic easy axis [100] direction at low temperatures, but switches to the uniaxial easy axis along $[1 \overline{1} 0]$ as the temperature increases to above $22 \mathrm{~K}$. The reorientation of the in-plane easy axis occurring at $22 \mathrm{~K}$ suggests that the variation of precessional phase originates from the reorientation of equilibrium magnetization via the giant MLD effect in ferromagnetic (GaMn)As film. To be specific, the reorientation of the easy axis from [100] to [110] direction occurring around $22 \mathrm{~K}$ results in a change of the relative angle between the probe polarization orientation and the equilibrium direction of magnetization. When 
temperature is below $22 \mathrm{~K}$, the cubic anisotropy is dominant(the cubic anisotropy constant is larger than the uniaxial anisotropy constant, $K_{u}<-K_{c}$ ), and thus the equilibrium direction of $\mathbf{M}$ lies in between [100] and [1 $\overline{10}]$, as determined by $\cos \left(2 \varphi_{M}\right)=-K_{\mathrm{u}} / K_{c}$, where $\varphi_{M}$ is the equilibrium magnetization orientation relative to the uniaxial easy axis [1 $\overline{10}][29,30]$. Based on our quantitative evaluation of the anisotropy fields at different temperatures [31], the equilibrium orientation of $\mathbf{M}\left(\varphi_{M}\right)$ is determined to be $4^{\circ}$ away from the [100] direction [see Fig.3(c)] at low temperature. Then one can easily obtain that $\varphi_{M^{-}} \beta<0$. When the temperature is increased above $22 \mathrm{~K}$, the equilibrium orientation of $\mathbf{M}$ jumps to the uniaxial easy axis [1 $\overline{10}$ ] direction due to $K_{u}>$ $-K_{c}[29,30]$, the equilibrium magnetization orientation approaches to $45^{\circ}$ with respect to [100] direction, thus $\varphi_{M}-\beta>0$. Because the precessional phase $\Phi\left(\varphi_{M}, \beta\right)$ is the function of both $\varphi_{M}$ and $\beta$, a dynamical phase reversal is thus observed with the sign change of $\varphi_{M}-\beta$. This dynamic phase reversal results from the competition of cubic and uniaxial magnetocrystalline anisotropy, thus showing that the phase of dynamic precession can be used to characterize the status of the magnetization vector relative to the magnetocrystalilne anisotropy fields.

Similarly, the dynamic phase reversal can also be driven by applying an external magnetic field. In this case, as shown in Fig.4(a), the phase of dynamic precession can again be reversed from negative to positive by magnetization switch due to the giant MLD effect. Here the orientation of the probe polarization is again fixed at $\beta \sim 40^{\circ}$ to maximize the MLD response at $8 \mathrm{~K}$, and the external magnetic field is applied in the plane of the sample along the [1] 0 ] crystallographic direction. As discussed above, the equilibrium orientation of $\mathbf{M}$ (given by $\varphi_{\mathrm{M}}$ ) is only $4^{\mathrm{o}}$ away from the cubic easy axis [100] at low external field [see Fig.4(b)], resulting in a 
negative sign of $\Phi\left(\varphi_{M}-\beta\right)$, as shown in Fig.4(a). Increasing of the external magnetic field then drives the magnetization reversal, which results in $90^{\circ}$ jumps of the magnetization between the four equivalent cubic easy axes of compressively-strained (Ga,Mn)As at low temperatures, which has been thoroughly studied previously[32-37]. Thus the magnetization will jump from the initial cubic easy axis along the [100] direction to the energetically-equivalent cubic easy axis [0 10] direction as the external magnetic field increases from 0 to $30 \mathrm{mT}$. Since the magnetic free energy of an in-plane magnetized (Ga,Mn)As sample can be described by the biaxial and uniaxial anisotropy energies as well as a Zeeman term [38], the magnetization orientation is determined by the competing effects of cubic and uniaxial anisotropies when the external magnetic field is much smaller than the anisotropy fields. For our case, we obtain the equilibrium orientation of $\mathbf{M}$ to be $\varphi_{M}=4^{\circ}$ away from cubic easy axis [0 $\left.\overline{1} 0\right]$ direction (i.e., $86^{\circ}$ away from the [100] direction), as illustrated in Fig.4(b). This new magnetization orientation then leads to a positive value of precession phase $\Phi\left(\varphi_{M}, \beta\right)$, and thus a positive MLD response, as shown in Fig.4(a). Although the orientation of magnetization will deviate somewhat from the easy axis $[0 \overline{1}$ 0] towards the external field direction of [1ํㅣ as the external field increases, the overall polarization rotation $\Delta \beta$ of the MLD response will remain positive, since $\varphi_{\mathrm{M}}$ remains within $45^{\circ} \leq \varphi_{M} \leq 86^{\circ}$ with further increases of the field, as shown in Fig.4(a). The observed dynamic phase reversal occurring for large MLD and small reversal switching fields demonstrates an efficient method for modulating the collective spin dynamics in (Ga,Mn)As, a feature of direct interest towards for applications in high-speed magnetic information storage.

\section{Conclusions}


In summary, the process of dynamic phase reversal of magnetization precession in ferromagnetic $(\mathrm{Ga}, \mathrm{Mn}) \mathrm{As}$ has been investigated by time-resolved pump-probe magneto-optical measurements based on the giant MLD effect. The observed dynamic phase reversal process is revealed to be sensitive to the modulation of the effective magnetic fields by varying either the temperature or the external magnetic field with linearly-polarized light excitation. Because the observed phenomenon does not require spin-polarized injection or additional fitting parameters, it provides an efficient and sensitive method to visualize the modulation of magnetization reorientation in ultrafast spin dynamics, and issue of direct interest for potential (Ga,Mn)As-based spintronic devices.

\section{Acknowledgments}

This work was supported by the National Basic Research Program of China (Nos. 2011CB922200, 2013CB922303), and by the National Natural Science Foundation of China (No. 10974195). The work at Notre Dame was supported by the National Science Foundation Grant DMR14-00432. 


\section{References:}

[1] B. Koopmans, M. van Kampen, W. J. M. de Jonge, J. Phys.:Condens. Matter 15 (2003) S723.

[2] H. Ohno, Science 281 (1998) 951.

[3] T. Jungwirth, JairoSinova, J. Mašek, J. Kučera, A. H. MacDonald, Rev.Mod. Phys. 78 (2006) 809.

[4] A. Kirilyuk, A.V. Kimel, T. Rasing, Rev. Mod. Phys., 82 (2010), p.2731.

[5] J. Wang, I. Cotoros, K. M. Dani, X. Liu, J. K. Furdyna, D. S. Chemla, Phys. Rev. Lett. 98 (2007) 217401.

[6] Y. Hashimoto, S. Kobayashi, H. Munekata, Phys. Rev. Lett. 100 (2008) 067202.

[7] Wang, D. M., Y. H. Ren, X. Liu, J. K. Furdyna, M. Grimsditch, R. Merlin, Phys. Rev. B 75 (2007) 233308.

[8] Van Kampen, M., C. Jozsa, J. T. Kohlhepp, P. LeClair, L. Lagae, W. J. M. de Jonge, B. Koopmans, Phys. Rev. Lett. 88 ( 2002) 227201.

[9] J. Chovan, I. E. Perakis, Phys. Rev. B 77 (2008) 085321.

[10] P. Němec, E. Rozkotová, N. Tesařová, F. Trojánek, E. De Ranieri,K. Olejník, J. Zmen, V. Novák, M. Cukr, P. Malý, T. Jungwirth, Nat. Phys.8 (2012) 411.

[11] M. Dobrowolska, K. Tivakornsasithorn, X. Liu, J. K. Furdyna, M. Berciu, K. M. Yu, W. Walukiewicz, Nat. Mater. 11 (2012) 444.

[12] N. Kazantseva, D. Hinzke, U. Nowak, R. W. Chantrell, U. Atxitia, O. Chubykalo-Fesenko, Phys. Rev. B 77(2008) 184428.

[13] U. Atxitia, O. Chubykalo-Fesenko, R. W. Chantrell, U. Nowak, A. Rebei, Phys. Rev. Lett. 
$102(2009) 057203$.

[14] J. Qi, Y. Xu, A. Steigerwald, X. Liu, J. K. Furdyna, I. E. Perakis, N. H. Tolk, Phys. Rev. B 79 (2009) 085304.

[15] A.Oiwa, H. Takechi, H. Munekata, J. Supercond. Nov. Magn. 18 (2005) 9.

[16] A. V. Kimel, G. V. Astakhov, A. Kirilyuk, G. M. Schott, G. Karczewski, W. Ossau, G.

Schmidt, L. W. Molenkamp, T. Rasing, Phys. Rev. Lett. 94 (2005) 227203.

[17] N. Tesařová, P. Tesařová, E. Rozkotová, J. Sŭbrt, H. Reichlová, D. Butkovičová, F. Trojánek,

P. Malý, V. Novák, T. Jungwirth, Appl. Phys. Lett. 100 (2012) 102403.

[18] N. Tesařová, J. Šubrt, P. Malý, P. Němec, C. T. Ellis, A. Mukherjee, J. Cerne, Rev. Sci. Instrum. 83 (2012) 123108.

[19] N. Tesařová, T. Ostatnický, V. Novák, K. Olejník, J. Šubrt, H. Reichlová, C. T. Ellis, A.

Mukherjee, J. Lee, G. M. Sipahi, J. Sinova, J. Hamrle, T. Jungwirth, P. Němec, J. Cěrne, K.

Výborny, Phys. Rev. B. 89 (2014) 085203.

[20] J. Qi, Y. Xu, N. H. Tolk, X. Liu, J. K. Furdyna, I. E. Perakis, Appl. Phys. Lett. 91 (2007)

112506; J. Qi, Y. Xu, A. Steigerwald, X. Liu, J. K. Furdyna, I. E. Perakis, N. H. Tolk, Phys. Rev.

B 79(2009) 085304; E. Rozkotová, P. Němec, N. Tesařová, P. Malý, V. Novák,

K. Olejník, M. Cukr, T. Jungwirth, Appl. Phys. Lett. 93 (2008) 232505.

[21] N. Tesařová, E. Rozkotová, H. Reichlová, P. Malý, V. Novák, M. Cukr, T. Jungwirth, P.

Němec, J. Nanosci. Nanotechnol. 12 (2012) 7477.

[22] E. Rozkotová, P. Němec, P. Horodyská, D. Sprinzl,F. Trojánek, P. Malý, V. Novák, K.

Olejník, M. Cukr, T. Jungwirth, Appl.Phys.Lett. 92 (2008) 122507. 
[23] J. Wang, Ł. Cywiński, C. Sun, J. Kono, H. Munekata, L. J. Sham, Phys. Rev. B 77 (2008) 235308.

[24] A. J. Lochtefeld, M. R. Melloch, J. C. P. Chang, E. S. Harmon, Appl. Phys. Lett. 69 (1996) 1465.

[25] G. V. Astakhov, R. I. Dzhioev, K. V. Kavokin, V. L. Korenev, M. V. Lazarev, M. N. Tkachuk, Yu. G. Kusrayev, T. Kiessling, W. Ossau, L. W. Molenkamp, Phys. Rev. Lett. 101 (2008)076602. [26] M. I. Dýakonov, V. I. Pereĺ, in Optical Orientation, edited by F. Meier and B. P. Zakharchenya, Elsevier, Amsterdam(1984d) Chap. 2, pp. 11-71.

[27] M. Sawicki, K.-Y. Wang, K. W. Edmonds, R. P. Campion,C. R. Staddon,N. R. S. Farley, C. T. Foxon, E. Papis, E. Kamiñska, A. Piotrowska, T. Dietl, B. L. Gallagher, Phys. Rev. B 71 (2005) 121302.

[28] J. Zemen, J. Kučera, K. Olejník, T. Jungwirth, Phys. Rev. B 80 (2009) 155203.

[29] U.Welp, V. K. Vlasko-Vlasov, X. Liu, J. K. Furdyna, T.Wojtowicz, Phys. Rev. Lett. 90 (2003) 167206.

[30] K. Wang, PhD Thesis, The University of Nottingham 133, 2005.

[31] H. Li, X. Liu, Y. Zhou, J.K. Furdyna, X. Zhang, Phys. Rev. B 91 (2015) 195204.

[32] B. Al-Qadi, N. Nishizawa, K. Nishibayashi, M. Kaneko, H. Munekata, Appl. Phys. Lett. 100 (2012) 222410 .

[33] G. P. Moore, J. Ferré, A. Mougi, M. Moreno, L. Daweritz, J. Appl.Phys. 94 (2003) 4530.

[34] J. Aoyama, Y. Hashimoto, S. Kobayashi, H. Munekata, J. Appl. Phys. 105 (2009) 07C518.

[35] G. V. Astakhov, A. V. Kimel, G. M. Schott, A. A. Tsvetkov, A. Kirilyuk, D. R. Yakovlev, G. 
Karczewski, W. Ossau, G. Schmidt, L. W. Molenkamp, T. Rasing, Appl. Phys. Lett. 86 (2005) 152506.

[36] J. Wang, I. Cotoros, D. S. Chemla, X. Liu, J. K. Furdyna, J. Chovan, I. E. Perakis, Appl. Phys. Lett. 94 (2009) 021101.

[37] Y. G. Zhu, X.H. Zhang, T. Li, X. Huang, L.F. Han, J. H. Zhao, Appl. Phys. Lett., 95 (2009) 052108.

[38] H. X. Tang, R. K. Kawakami, D. D. Awschalom, M. L. Roukes, Phys. Rev. Lett. 90 (2003) 107201. 


\section{Figure Captions:}

Fig.1 (color online) (a)Temporal profiles of Kerr rotations measured at $10 \mathrm{~K}$ and $1.58 \mathrm{eV}$ for circularly- and linearly-polarized optical pumping without an external magnetic field. The inset shows that the precession response vanishes at $80 \mathrm{~K}$ regardless of polarization of the pumping beam. The strong dependences of the Kerr responses on polarization are confined to the shadow region. The solid line is a single exponential decay fitting result.

Fig. 2 (color online) The magnetic precession response at different polarization orientations of the probe beam, excited by linearly-polarized light at $1.58 \mathrm{eV}$ and at a power density of $2.33 \mu \mathrm{Jcm}^{-2}$ with no external magnetic field applied. The angles refer to the directions of probe polarization relative to the $[100]$ axis.

Fig. 3(color online) (a) Temperature dependence of the magnetic precession response induced by linearly-polarized $1.58 \mathrm{eV}$ light pulsewith no external magnetic field. The pumping power density is $2.33 \mu \mathrm{Jcm}^{-2}$; (b) Temperature dependence of the remnant magnetic moment in the annealed $\mathrm{Ga}_{0.964} \mathrm{Mn}_{0.036} \mathrm{As}$ film along the cubic easy axis [100], the uniaxial easy axis [1 10$]$, and the uniaxial hard axis [110] directions. The dominant magnetic anisotropy switches from cubic to uniaxial behavior at $T=22 \mathrm{~K}$; (c)Schematic of the equilibrium magnetization reorientation from near $[100]$ to $[1 \overline{1} 0]$ directions occurring around $22 \mathrm{~K}$ with the probe polarization orientation $\beta$ fixed (depicted as the red arrow line). Initial and final equilibrium magnetization orientations are marked as $\mathbf{M}_{\mathrm{i}}$ and $\mathbf{M}_{\mathrm{f}}$, respectively. 
Fig.4 (color online) (a) Magnetic precession response excited by linearly-polarized light at $8 \mathrm{~K}$ and $1.58 \mathrm{eV}$, and observed at several external magnetic fields applied along the [1 $1 \overline{1} 0]$ directions. (b) Schematic of the four-state magnetization switchings between the four equivalent cubic easy axes as the field is increased. (c) Schematic of the equilibrium magnetization reorientation with an applied magnetic field, causing the sample to be initially magnetized along $\mathbf{M}_{\mathrm{i}}$, and then to jump to the $\mathbf{M}_{\mathrm{f}}$ direction as the field is increased, and eventually to rotate towards the [1 10$]$ direction when the field is larger than the anisotropy field. The red arrow depicts the polarization orientation of probe beam. 

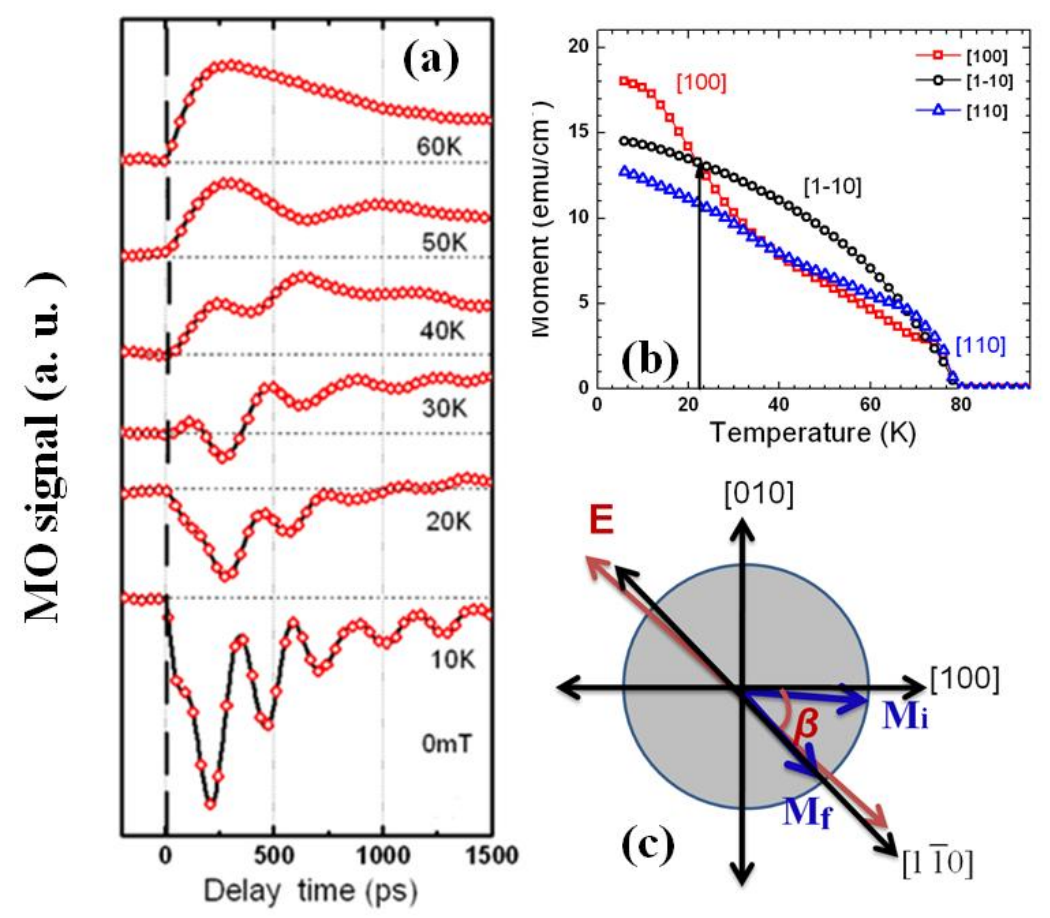
Figure 2

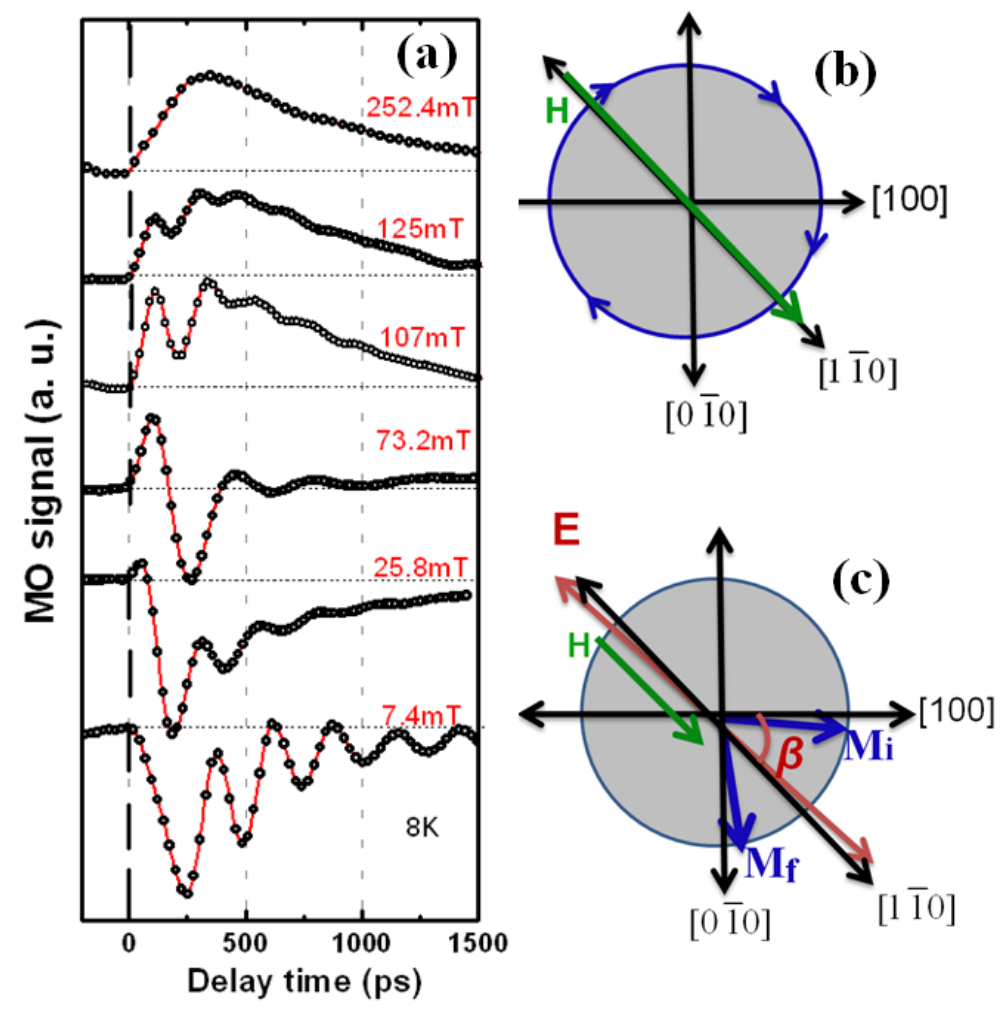


Figure 3

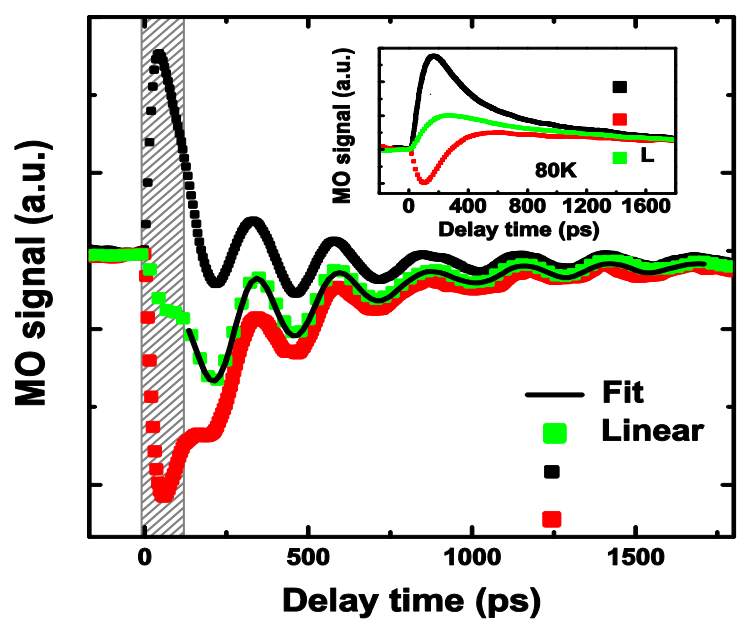

Linear

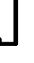

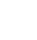


